\title{
Characterization and interaction of driving factors in karst rocky desertification: a case study from Changshun, China
}

\author{
E. Q. Xu ${ }^{1,2}$ and H. Q. Zhang ${ }^{1}$ \\ ${ }^{1}$ Key Laboratory of Land Surface Pattern and Simulation, Institute of Geographical Sciences and Natural Resources \\ Research, Chinese Academy of Sciences, Beijing 100101, PR China \\ ${ }^{2}$ University of Chinese Academy of Sciences, Beijing 100049, PR China \\ Correspondence to: H. Q. Zhang (zhanghq@igsnrr.ac.cn)
}

Received: 9 September 2014 - Published in Solid Earth Discuss.: 9 October 2014

Revised: 12 November 2014 - Accepted: 19 November 2014 - Published: 16 December 2014

\begin{abstract}
As the most severe ecological issue in southwest China, karst rocky desertification (KRD) has both threatened and constrained regional sustainable development. Comprehensively understanding the relationship between the evolution of KRD and relevant driving data would provide more information to combat KRD in such complex karst environments. Past studies have been limited in quantifying the relative importance of driving factors influencing fine-scale KRD evolution, and have also lacked insight into their interactive impacts. To address these issues, we have used geographical information system techniques and a geographical detector model to explore the spatial consistency of driving factors and their interactions in relation to the evolution of KRD. Changshun County in China was selected as a representative area for the study. Nine relevant driving factors, including both natural and anthropogenic factors, were studied in regard to their relationships with KRD transformation between 2000 and 2010. Our results demonstrate the relative importance of driving data in influencing the improvement and deterioration of KRD. Lithology, soil type and road influence are identified as the leading factors. Interestingly, to our study at least, there is no significant difference between the impacts of natural and anthropogenic factors influencing KRD improvement, and even natural factors have a higher impact on KRD deterioration. Factors were found to enhance the influence of each other for KRD transformation. In particular, the results show a non-linearly enhanced effect between driving factors, which significantly aggravates KRD. New information found in our study helps to effectively control and restore areas afflicted by KRD.
\end{abstract}

\section{Introduction}

China has approximately $3.44 \times 10^{6} \mathrm{~km}^{2}$ of karst areas, about $36 \%$ of its total land, and $15.6 \%$ of all the $22 \times 10^{6} \mathrm{~km}^{2}$ karst areas in the world (Jiang et al., 2014). The continuously distributed karst region, which is mostly located in eight provinces of southwestern China (Guizhou, Yunnan, Guangxi, Chongqing, Sichuan, Hunan, Hubei, and Guangdong) is one of the world's most extensive and welldeveloped karst landscapes (Wang et al., 2004b; Xu et al., 2013). Karst rocky desertification (KRD) has been identified as the most severe ecological issue threatening and constraining southwestern China (Bai et al., 2013; Wang et al., 2004b). The KRD is a process of land degradation involving serious soil erosion, extensive exposure of basement rocks, drastic decrease in soil productivity, and the appearance of a desertlike landscape (Wang et al., 2004b). Therefore, the National Reform and Development Commission commenced implementation of a pilot KRD restoration project for 100 counties in the region in 2008 and expanded the project to 200 counties in 2011.

The restoration and reconstruction projects have been effective (Qi et al., 2013; Zeng et al., 2007), but KRD improvement can easily reverse in local areas (Sheng et al., 2013). The mutual transformation (improvement or deterioration) of different types of KRD land has been remarkable (Bai et al., 2013), perhaps because KRD occurs as a result of multiple factors including the inherent natural background and intensive human activities in the complex karst environment (Jiang et al., 2014; Liu et al., 2008b; Wang et al., 2004b; Yang et al., 2011). The lack of insight into the joint impacts 


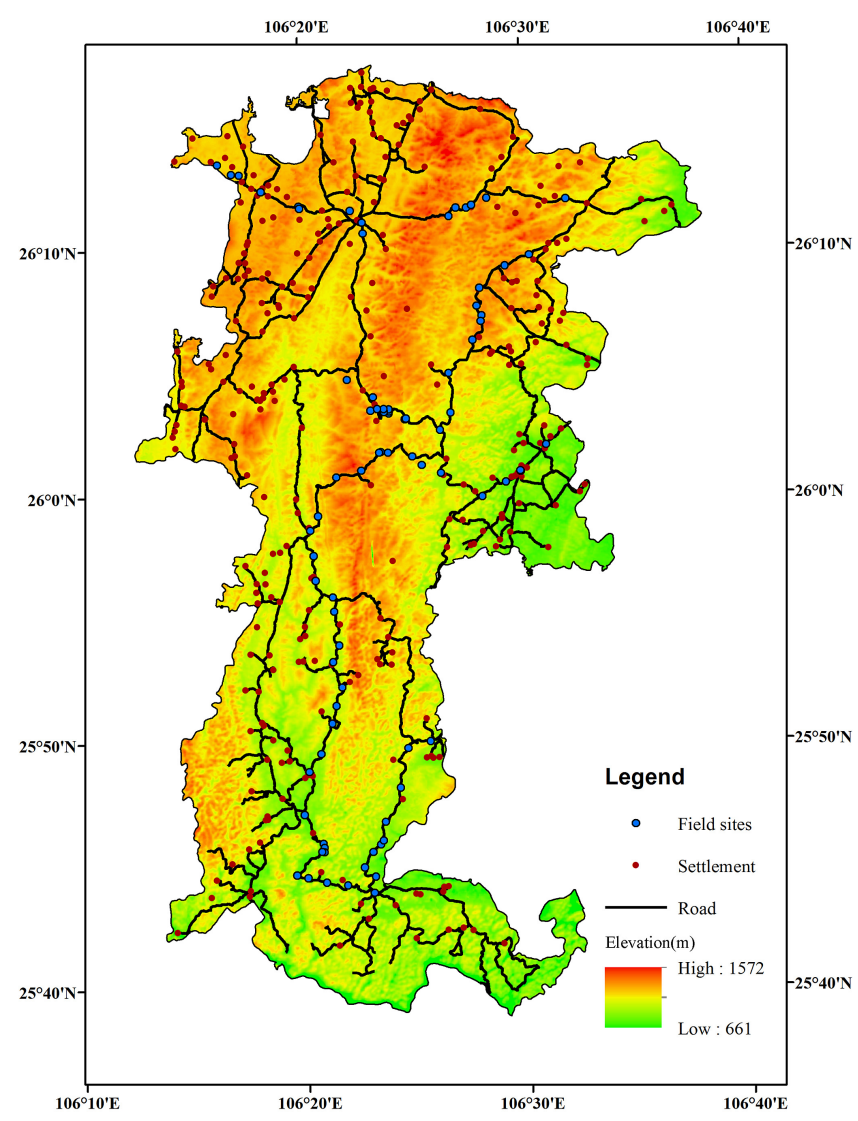

Figure 1. The location of Changshun County.

of driving factors on KRD evolution can affect restoration efforts. To control, manage and restore KRD, analyses of how relative driving factors influence the evolution of KRD and determinations of their corresponding contributions are essential.

Previous studies have undertaken analyses of different driving factors on KRD based on measurable physical data and socioeconomic census data. The evolution of KRD is associated with a considerable number of natural factors, e.g., meteorological factors including temperature and precipitation (Peng and Wang, 2012; Xiong et al., 2009), topographic factors consisting of the elevation, slope (Huang and Cai, 2007; Jiang et al., 2009) and aspect (Zhou et al., 2007), and lithological factors (i.e., types of carbonate rock assemblages) (Li et al., 2009b; Wang et al., 2004a). Human activities, such as farming on hilly land, overgrazing, and felling of forest and shrubs for cooking (Jiang et al., 2014; Li et al., 2009b, 2008a; Wu et al., 2011; Yan and Cai, 2013), have also recently become pivotal factors in KRD. The natural and anthropogenic factors above jointly influence the evolution of KRD in southwestern China.

Few previous studies have discussed the relative importance of driving factors contributing to the evolution of KRD (Huang and Cai, 2007; Jiang et al., 2009, 2014; Li et al.,
Table 1. Landsat image description.

\begin{tabular}{cccc}
\hline Time & Path & Row & Date \\
\hline 2000 & 127 & 42 & 4 Nov 2000 \\
2010 & 127 & 42 & 31 Oct 2010 \\
\hline
\end{tabular}

2009b; Peng and Wang, 2012; Xiong et al., 2009; Yan and Cai, 2013). Impacts of driving factors have been analyzed separately, and the leading driving factors have been qualitatively or semi-quantitatively evaluated. A limited number of studies have used regression modeling (Liu et al., 2008a), factor analysis (Li et al., 2009a), or redundancy analysis (Yang et al., 2011) to study such relationships. These studies were constrained at the scale of a county or regions (e.g., the third or fourth administrative unit in China in terms of scale), and were missing any spatially consistent information on KRD evolution as a result of driving factors. Additionally, consideration of the interaction of different factors is lacking in previous work. All of these factors are necessary for a deeper understanding of the problem. Fortunately, a geographical detector model (Li et al., 2013; Wang et al., 2010b), that can calculate the relative importance of various factors, provides an opportunity to consistently quantify more spatial information on the driving factors and their interactions with regard to the evolution of KRD.

The objective of this study is to investigate the relationships between the spatial evolution of KRD and its driving factors at a fine scale. Based on a geographical detector model, we have quantified the relative importance of different natural and anthropogenic factors. Furthermore, we have identified the leading factors and analyzed their interactive impacts on both the improvement and deterioration of KRD.

\section{Materials and methods}

\subsection{Study area}

Changshun County is located in central Guizhou Province in China, roughly between $106^{\circ} 13^{\prime} 06^{\prime \prime}$ and $106^{\circ} 38^{\prime} 48^{\prime \prime} \mathrm{E}$, and $25^{\circ} 38^{\prime} 48^{\prime \prime}$ and $26^{\circ} 17^{\prime} 30^{\prime \prime} \mathrm{N}$ (Fig. 1). The county covers an area of $1552 \mathrm{~km}^{2}$ within a karst peak-cluster depression landscape that contains a combination of karst cones and depressions between cones. It lies within the subtropical monsoon climate zone with a mean annual temperature of $14^{\circ}$ to $14.5^{\circ} \mathrm{C}$ and a mean annual precipitation of 1160 to $1355 \mathrm{~mm}$ (Xu et al., 2013). Its elevation is between 661 and $1572 \mathrm{~m}$. Changshun is one of the areas most affected by KRD in Guizhou, with nearly $30 \%$ of its area impacted (Wang, 2010). 


\subsection{Data description}

\subsubsection{Satellite data and preprocessing}

An Enhanced Thematic Mapper Plus (ETM+) image was chosen to map the KRD of Changshun County in 2000 and 2010. The 2010 image was collected as a strip-repaired ETM+ image, provided by the International Scientific Data Service Platform, Computer Network Information Center, Chinese Academy of Sciences (http://www.gscloud.cn/). Table 1 presents the details of the paths, rows and acquisition dates for Changshun.

ETM+ image was preprocessed by applying geometric and atmospheric corrections. A digital elevation model (DEM) with 30 ground control points, which were taken from 1: 50000 topographic maps (provided by the Institute of Geographic Sciences and Natural Resources Research, Chinese Academy of Sciences), was incorporated in the geometric corrections. This resulted in a root-mean-square spatial positioning error of less than 0.5 pixels for each image. The FLAASH module from the Environment for Visualizing Images (ENVI) software (Solutions, 2009) was used for atmospheric corrections.

\subsubsection{Categorizing and quantifying karst rocky desertification}

Based on mapping of bedrock exposures, vegetation and soil coverage (Li et al., 2009b), karst areas can be classified as no KRD, potential KRD, slight KRD, moderate KRD, severe KRD and extremely severe KRD (Table 2). The distribution of KRD in Changshun County in 2000 and 2010 was mapped by visual interpretation. In 2010, 86 field measurements were made to assess the accuracy of the method in Changshun (Fig. 1). The accuracy was $90.7 \%$ (Xu et al., 2013).

To use the geographical detector model (Li et al., 2013; Wang et al., 2010b), the evolution of KRD needs to be quantified as a measurable index. Because the KRD classification results are categorical variables, we assigned the six KRD categories as $6 \mathrm{KRD}_{i}$ levels (where $i$ ranges from 1 to 6 , representing the six increasing levels of KRD intensity: no $\mathrm{KRD}$, potential KRD, light KRD, moderate KRD, severe $\mathrm{KRD}$ and extremely severe KRD, respectively) (Table 2). $\mathrm{KRD}_{i}$ is the midpoint of bedrock rate for each KRD category (i.e., $\mathrm{KRD}_{1}=10, \mathrm{KRD}_{2}=25, \mathrm{KRD}_{3}=40, \mathrm{KRD}_{4}=$ $60, \mathrm{KRD}_{5}=80$ and $\mathrm{KRD}_{6}=95$ ).

Then evolution index of KRD for each raster can be calculated as follows:

$\mathrm{E}-\mathrm{KRD}=\mathrm{KRD}_{i}^{t_{0}}-\mathrm{KRD}_{j}^{t}$

where $t_{0}$ and $t$ denote the period, i.e., 2000 and 2010, respectively in this study; $i$ and $j$ range from 1 to 6 , representing the six levels of KRD intensity as above; $\mathrm{KRD}_{j}^{t_{0}}$ and $\mathrm{KRD}_{i}^{t}$ are measurable indices of KRD from Table 2 in 2000 and 2010, respectively. A positive E-KRD (improvement index) indicates that the KRD land improves as the bedrock rate of an area decreases; a negative E-KRD (deterioration index) indicates that the KRD land deteriorates. A larger absolute value of E-KRD signifies a more significant intensity of KRD transformation.

\subsubsection{Driving factors data sets}

We chose nine relevant driving factors to study their relationship with the evolution (improvement or deterioration) of KRD based on previous studies (Jiang et al., 2009; Li et al., 2009b; Wang et al., 2004a; Yang et al., 2011). Natural factors include soil type classified by the genetic soil classification of China (Shi et al., 2004), lithology, vegetation type, elevation and slope; human factors include road influences (buffer of roads), settlement influences (buffer of settlements), gross domestic product (GDP) density, and population density. It is difficult to quantify human activities as a detailed spatial unit. Therefore, we used geographic information system (GIS) techniques to quantify such land use information, including farming on hilly lands, overgrazing, felling of forest or shrubs for cooking, and KRD restoration projects. The buffer methods calculate the Euclidean distance to roads and settlements (i.e., road and settlement influence) as proxies of the distribution of human activities (Simpson and Christensen, 1997). A shorter distance denotes a greater human influence. The presence of roads corresponds to the utilization of natural resources (Yang et al., 2013), and the distribution of settlements is related to the range of residents' daily lives and the implementation of restoration projects (Yang et al., 2011). The detailed data sources and processing of the nine sets of driving data are listed in Table 3.

In the geographical detector model, continuous data should be transformed into discrete intervals (Cao et al., 2013a; Wang et al., 2010b). The discretization method depends on optimal classifications or prior knowledge (Li et al., 2008; Wang et al., 2010a). With the exception of the soil, lithology and vegetation data, the driving data can be considered to be continuous variables (Table 3 ). Based on the distribution and prior knowledge of data, we majorly used the method of natural breaks coupled with the professional knowledge and rounded the intervals as the integer format (Fig. 2). As continuous variables have local characteristics, natural breaks can seek to minimize each interval's average deviation from the interval mean while maximizing each interval's deviation from the means of the other groups. In other words, the method seeks to reduce the variance within intervals and maximize the variance between intervals. The discretization standards for the corresponding data are also shown in Fig. 2.

\subsection{Geographical detector model}

The geographical detector model is a new spatial analysis method used to assess the relationship between driving 
a)

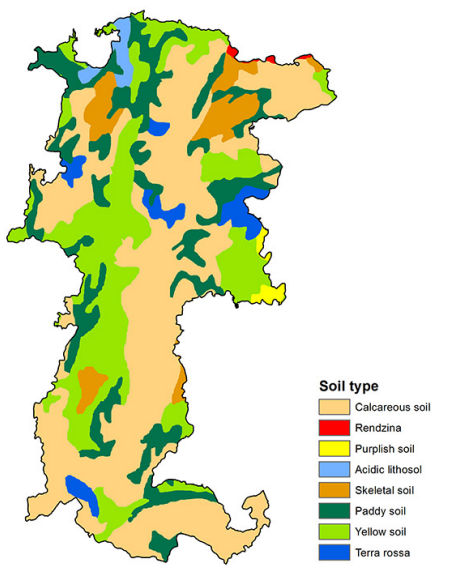

d)

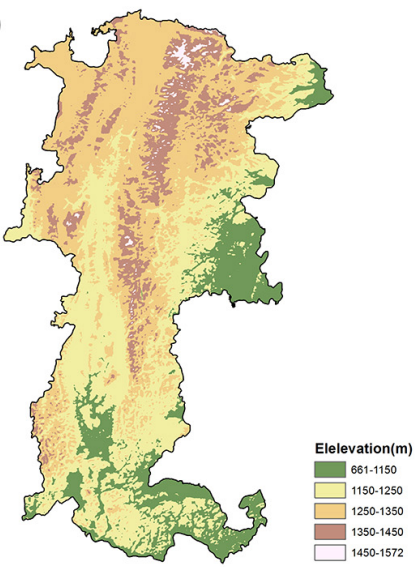

g)

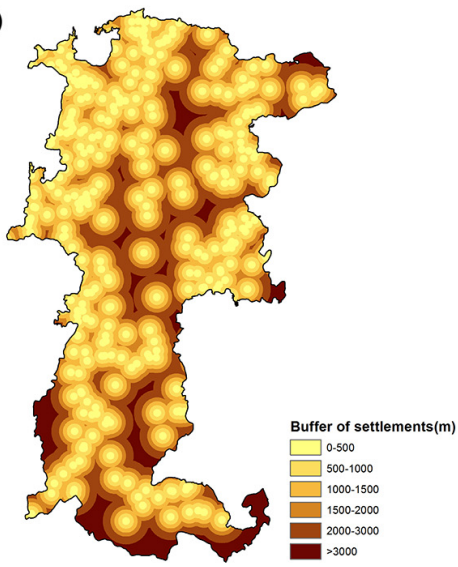

b)
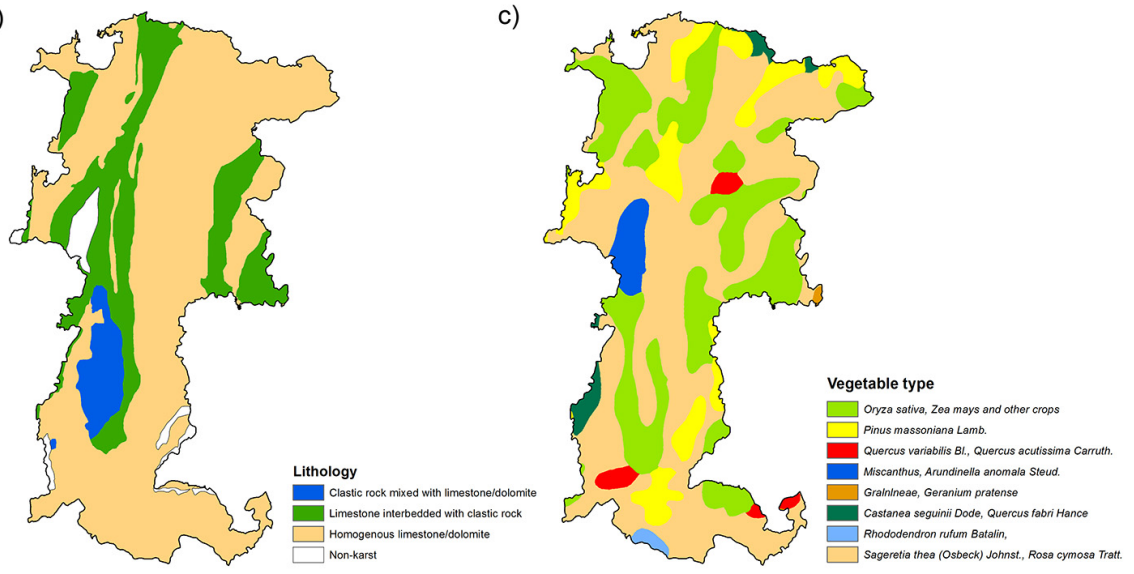

e)

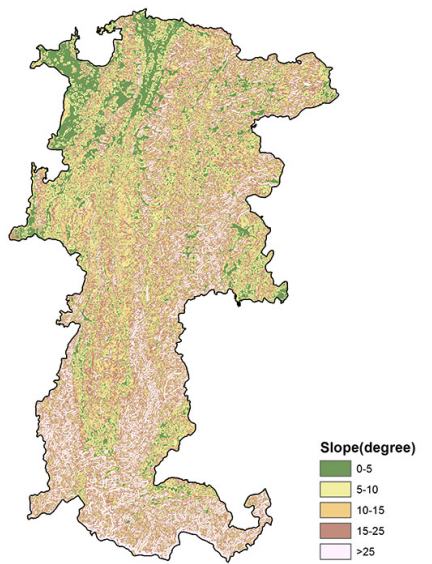

h)

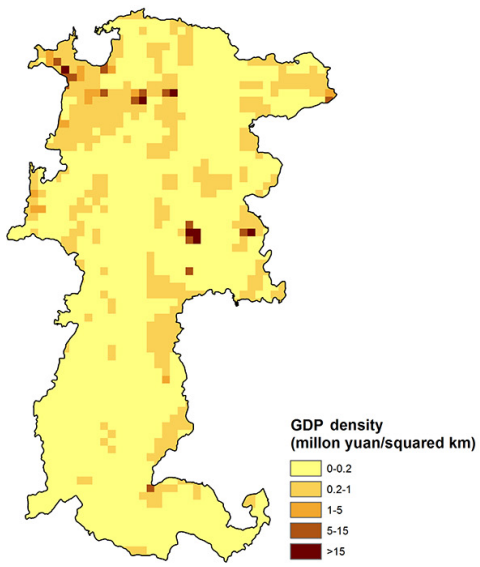

f)

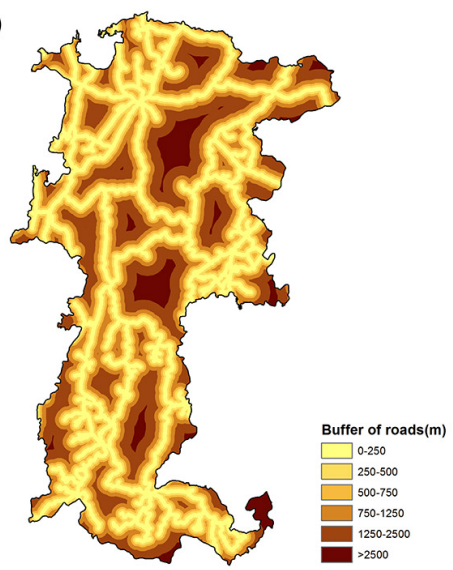

i)

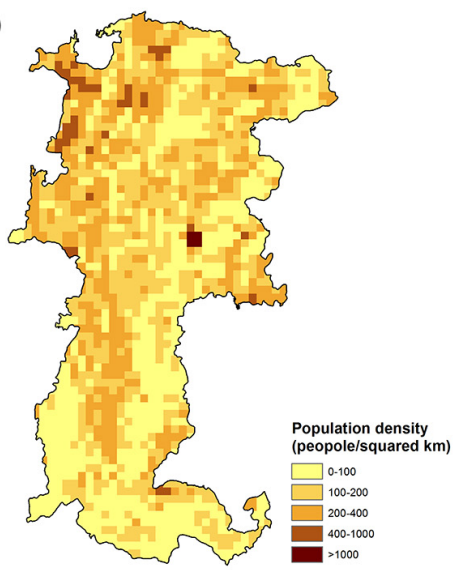

Figure 2. Driving database of the evolution of karst rocky desertification (a) soil type; (b) lithology; (c) vegetable type; (d) elevation; (e) slope; (f) road influence; (g) settlement influence; (h) gross domestic product density; (i) population density.

factors and relevant resultant outcomes (Hu et al., 2011; Li et al., 2013; Wang et al., 2010b). The model compares the spatial consistency of a resultant outcome distribution (e.g., the KRD in our study) to strata of driving factors based on a spatial variance analysis. It can extract information without any assumptions or restrictions with respect to the vari- ables. Also, it can be used for both quantitative and nominal data. The model can resolve the following four questions. (1) What is the geographical domain of the outcome? (2) What are the driving factors responsible for the outcome? (3) What is the relative importance of each driving factor? 
Table 2. Classification and quantification standard for karst rocky desertification.

\begin{tabular}{|c|c|c|c|}
\hline & $\begin{array}{l}\text { Bedrock exposure } \\
\qquad(\%)\end{array}$ & $\begin{array}{l}\text { Vegetation and soil } \\
\text { cover }(\%)\end{array}$ & $\mathrm{KRD}_{i}$ Index \\
\hline $\begin{array}{l}\mathrm{KRD}_{1} \text { : no karst rocky desertification } \\
(\text { No KRD) }\end{array}$ & $<20$ & $>80$ & 10 \\
\hline $\begin{array}{l}\mathrm{KRD}_{2} \text { : potential karst rocky desertification } \\
\text { (Potential KRD) }\end{array}$ & $20-30$ & $70-80$ & 25 \\
\hline $\begin{array}{l}\mathrm{KRD}_{3} \text { : light karst rocky desertification } \\
\text { (Light KRD) }\end{array}$ & $31-50$ & $50-69$ & 40 \\
\hline $\begin{array}{l}\mathrm{KRD}_{4} \text { : moderate karst rocky desertification } \\
\text { (Moderate KRD) }\end{array}$ & $51-70$ & $30-49$ & 60 \\
\hline $\begin{array}{l}\mathrm{KRD}_{5}: \text { severe karst rocky desertification } \\
\text { (Severe KRD) }\end{array}$ & $71-90$ & $10-29$ & 80 \\
\hline $\begin{array}{l}\text { KRD }_{6} \text { : extremely severe karst rocky } \\
\text { desertification (extremely severe KRD) }\end{array}$ & $>90$ & $<10$ & 95 \\
\hline
\end{tabular}

(4) Do driving factors operate independently or are they interconnected? (Wang et al., 2010b).

The power of determinant (PD) value and the interactive $\mathrm{PD}$ value are two novel indicators used to assess the relationship between outcomes and their driving factors in the geographical detector model (Li et al., 2013; Wang et al., 2010b). The geographical detector model overlays the distribution of K (e.g., E-KRD in our study) over several strata of driving factors of $\mathrm{D}$ (i.e., one of driving data). $D_{i}$, where $i=1,2, \ldots n$, and $n$ is the number of categorical types of $D$, are the discrete attributes associated with a stratum of driving factors of $D$, which is denoted as $D=\left\{D_{i}\right\}$ (Li et al., 2013; Wang et al., 2010b), then the study regions were divided to sub-regions $\left(D_{1}, D_{2}, \ldots D_{n}\right)$. The mean value and the dispersion variance of $K$ (denoted as $\sigma_{K_{D, i}}^{2}$ ) can be calculated by the model. Next, PD is calculated as follows:

$\mathrm{PD}=1-\frac{1}{N \times \sigma_{K}^{2}} \sum_{i=1}^{n} N_{D, i} \times \sigma_{K_{D, i}}^{2}$,

where $\mathrm{N}$ denotes the number of samples over the entire region of interest; $N_{D, i}$ is the number of samples in the subregion of $i$ category of $D ; \sigma_{K}^{2}$ denotes the variance of $\mathrm{K}$ over the entire region; the dispersion variance of $\mathrm{K}$ over the subregions of the attributes $D_{i}$ is denoted as $\sigma_{K_{D, i}}^{2}$. When the $\sigma_{K_{D, i}}^{2}$ of each subregion is small, the variances between subregions is large and the PD is large (which means that such a division explains most or even all of the spatial $K$ variation). Therefore, a higher PD indicates that the driving factor (D) has a larger impact on $K$. If the driving factor $(D)$ completely controls the total $K$, then $\mathrm{PD}=1$.

The package of geographical detector model used in our study is a version of Excel-GeoDetector (http://www. sssampling.org/Excel-GeoDetector/). All of the driving data and the E-KRD index were resampled in the WGS84 projection constrained by the same boundary of Changshun County in GIS. A pixel size of $100 \times 100 \mathrm{~m}$ was used to extract rel- evant data. Finally, all the data were extracted as input data for the Excel-GeoDetector.

\section{Results}

\subsection{Spatial evolution of KRD}

Figure 3 shows improvement and deterioration indices from 2000 to 2010 for Changshun County. Areas of KRD transformation are widely detected. The transformed area is $410 \mathrm{~km}^{2}$, including $301 \mathrm{~km}^{2}$ where the class improved and $109 \mathrm{~km}^{2}$ where it deteriorated. The improvement index ranges from 15 to 70 and the deterioration index ranges from -15 to -85 . Areas of KRD improvement and deterioration and their intensities show different spatial distribution characteristics. In general, significantly transformed areas (i.e., large absolute values of E-KRDs) are relatively small and concentrated in local areas. Areas of significant deterioration are mainly located in the townships of Weiyuan and Kaizuo. In comparison, KRD lands in western Changshun, including the townships of Baisuo and Yingpan, significantly improved from 2000 to 2010. Meanwhile, areas with small absolute values of E-KRDs, which indicate a relatively low intensity of KRD transformation, are widely distributed through the whole study area.

\subsection{Influence of driving factors on KRD improvement}

\subsubsection{Importance of driving factors influencing KRD improvement}

Using the geographical detector model, PDs of the nine aforementioned driving factors influencing KRD improvement index were calculated. Their order is as follows (the number in parentheses is the corresponding PD value): $\operatorname{lithology}(0.154)>\operatorname{road}(0.135)>$ soil $(0.120)$ 
a)

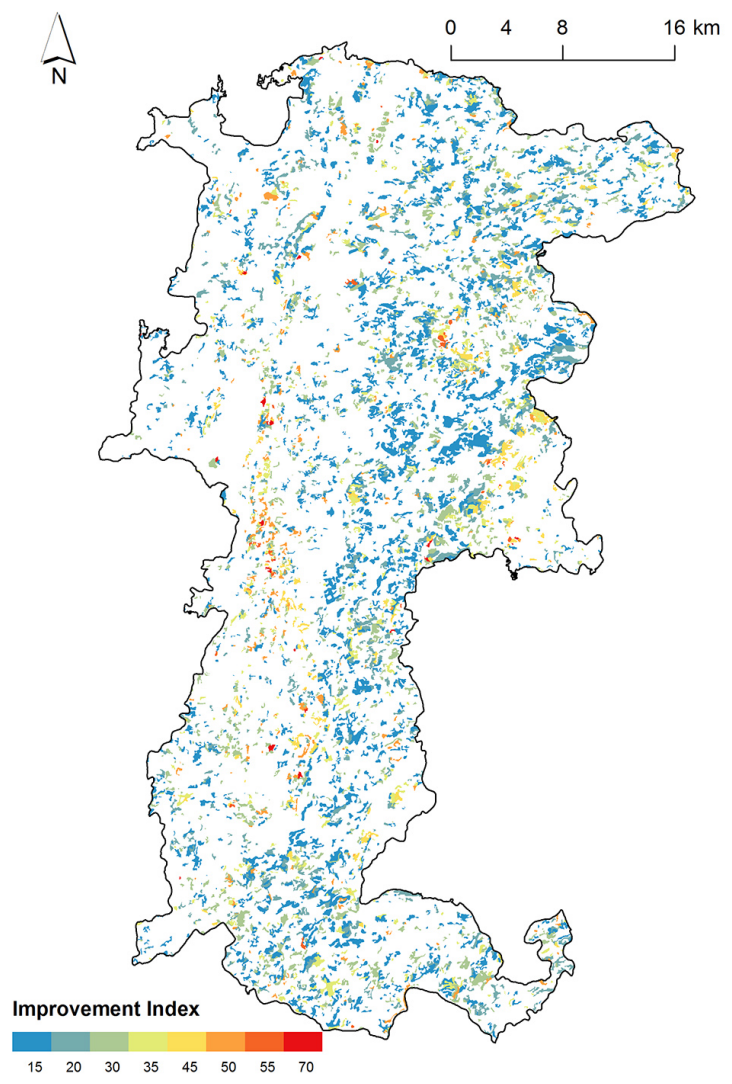

b)

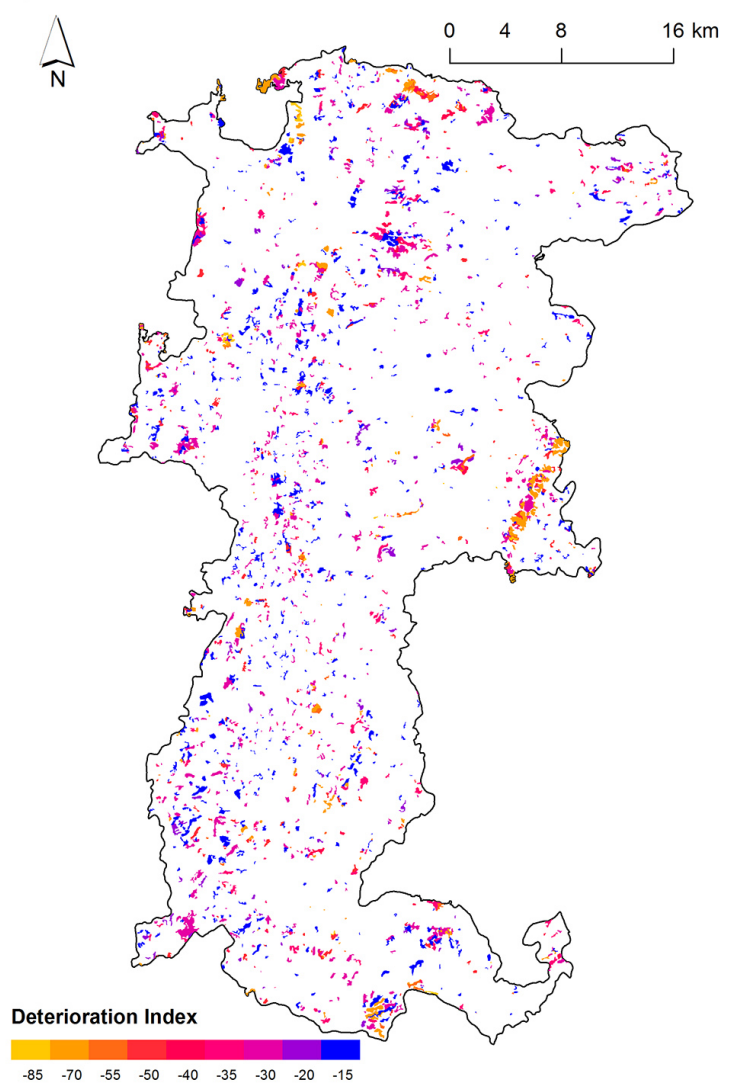

Figure 3. The spatial evolution index of karst rocky desertification from 2000 to 2010 (a) improvement index; (b) deterioration index.

$>$ population $(0.105)>$ vegetation $(0.088)>$ settlement $(0.073)>$ elevation $(0.062)>$ slope $(0.029)>$ GDP $(0.013)$.

The lithology, road influence, soil and population density (ranking first to fourth, respectively) are considered to be the major driving factors in Changshun. In contrast, the slope and GDP density have low relative importance when it comes to explaining the spatial distribution of KRD improvement. Considering the order of PDs, both natural and anthropogenic factors influence the improvement of KRD. This implies that natural factors (with ranks of first, third, fifth, seventh, and eighth) and anthropogenic factors (with ranks of second, fourth, sixth, and ninth) have similar overall impact. This information confirms that the effect of restoration project implementations would improve if the natural impact factors on the evolution of KRD were considered.

\subsubsection{Variations in KRD improvement corresponding to different driving factor levels}

The geographical detector model calculated mean values for the KRD improvement index for various levels of each driving factor, and indicated significant differences for all levels. The model helps to analyze how a particular driving factor influences KRD improvement. For example, the PD of lithology shows that it has the greatest impact. According to the model, the mean values of improvement indices for three lithological types show significant differences, and their ranks are as follows (with the number in brackets as the corresponding index value): clastic rock mixed with limestone/dolomite (32.6) > limestone interbedded with clastic rock (27.7) $>$ homogenous limestone/dolomite (22.3). These three lithological types show an increasing proportion of limestone or dolomite, which indicates that the proportion of limestone or dolomite is positively associated with improved intensities of KRD.

Our results also indicate that the distance to a road, as a proxy of human activity levels, influences the spatial distribution of KRD improvement. The order of mean index values for six levels of road influence (Table 4) suggests that the distance to a road is positively correlated to improved intensities of KRD. This means that a greater road influence (i.e., better access to roads) enhances the successful implementation of KRD restoration projects and accelerates the improvement of KRD. 
Table 3. Driving data sources and processing.

\begin{tabular}{|c|c|c|c|c|c|}
\hline Input data set & Data source & Format & Processing & Variable & \\
\hline Soil & $\begin{array}{l}\text { Institute of Geographic Sciences and Natural } \\
\text { Resources Research, Chinese Academy of Sciences }\end{array}$ & Polygon & Format transformation & Discrete & $1: 500000$ \\
\hline Lithology & Local map of Changshun County & Polygon & $\begin{array}{l}\text { Digitization and format } \\
\text { transformation }\end{array}$ & Discrete & $1: 500000$ \\
\hline Vegetation & $\begin{array}{l}\text { Institute of Geographic Sciences and Natural } \\
\text { Resources Research, Chinese Academy of Sciences }\end{array}$ & Polygon & Format transformation & Discrete & $1: 500000$ \\
\hline Elevation & $\begin{array}{l}\text { International Scientific Data Service Platform, } \\
\text { Computer Network Information Center, and } \\
\text { Chinese Academy of Sciences }\end{array}$ & Raster & Resampling & Continuous & $1: 50000$ \\
\hline Slope & $\begin{array}{l}\text { International Scientific Data Service Platform, } \\
\text { Computer Network Information Center, and } \\
\text { Chinese Academy of Sciences }\end{array}$ & Raster & $\begin{array}{l}\text { Generated from the } \\
\text { elevation map }\end{array}$ & Continuous & $1: 50000$ \\
\hline Buffer of roads & Local map of Changshun County in 2000 & Polyline & $\begin{array}{l}\text { Digitization and buffer } \\
\text { analysis }\end{array}$ & Continuous & $1: 50000$ \\
\hline $\begin{array}{l}\text { Buffer of } \\
\text { settlements }\end{array}$ & Local map of Changshun County in 2000 & Point & $\begin{array}{l}\text { Digitization and buffer } \\
\text { analysis }\end{array}$ & Continuous & $1: 50000$ \\
\hline Gross Domestic & Data Center for Resources and Environmental & Raster & Resampling & Continuous & $1: 500000$ \\
\hline Product density & Sciences, Chinese Academy of Sciences & & & & \\
\hline $\begin{array}{l}\text { Population } \\
\text { density }\end{array}$ & $\begin{array}{l}\text { Data Center for Resources and Environmental } \\
\text { Sciences, Chinese Academy of Sciences }\end{array}$ & Raster & Resampling & Continuous & $1: 500000$ \\
\hline
\end{tabular}

Table 4. Difference of mean improvement KRD index between levels of road influence.

\begin{tabular}{lcccccc}
\hline & Level 1 & Level 2 & Level 3 & Level 4 & Level 5 & Level 6 \\
\hline Level 1 & & & & & & \\
Level 2 & Y & & & & & \\
Level 3 & Y & Y & & & & \\
Level 4 & Y & Y & N & & & \\
Level 5 & Y & Y & Y & Y & & \\
Level 6 & Y & Y & Y & Y & N & \\
\hline
\end{tabular}

Level 1: 0-250 m; level 2: 250-500 m; level 3: 500-750 m; level 4: 750-1250 m; level 5:

1250-2500 m; level 6: $>2500 \mathrm{~m}$. "Y" denotes that the difference of influence between the two factor levels is significant with the confidence of $95 \%$; and " $\mathrm{N}$ " denotes that it is not.

The order of mean values for six road influence levels of improvement index is as follows: level 2 $(27.1)>$ level $1(26.3)>\operatorname{level} 3(24.0) \approx \operatorname{level} 4(22.6)>\operatorname{level} 5(20.6) \approx \operatorname{level6} 6(20.1)$. “ $\approx$ ” denotes that there is no significant difference between two levels (the same meaning is the following content).

\subsubsection{Interactions of driving factors influencing KRD improvement}

The interactive impact of two factors $(A$ and $B)$ on KRD improvement generally differs from the simple linear summation of the separate impacts of $A$ and $B$, i.e., PD $(A \cap B) \neq \mathrm{PD}(A)+\mathrm{PD}(B)$. PDs of their interactive impacts were calculated by the geographical detector model. The top 10 PDs of interactive impacts, which range from 0.205 to 0.283 , are greater than the highest PD of a single factor (i.e., PD (lithology) $=0.150)$ (Table 5). This shows that the combined impact of factors on KRD improvement would be greater than single factors, i.e., $\mathrm{PD}(A \cap B)>\mathrm{PD}(A)$ or $\mathrm{PD}$ $(B)$. Moreover, the road influence associated with vegetation and the elevation associated with population density reflect a non-linearly enhanced impact on the KRD improvement, i.e., $\mathrm{PD}(A \cap B)>\mathrm{PD}(A)+\mathrm{PD}(B)$.

\subsection{Influence of driving factors on KRD deterioration}

\subsubsection{Importance of driving factors influencing KRD deterioration}

The PDs of the nine driving factors influencing the KRD deterioration index were calculated. Their order is as follows: soil $(0.194)>$ lithology $(0.151)>$ road $(0.143)>$ settlement $(0.140)>$ elevation $\quad(0.138)>$ vegetation $\quad(0.099)>$ slope $(0.056)>\operatorname{GDP}(0.022)>$ population $(0.014)$.

This order differs from that of the KRD improvement. Except for the settlement influence (rank fourth), the lithology, soil and road influence (rank first, second and third, 
Table 5. Interaction between pairs of driving factors influencing the KRD improvement.

\begin{tabular}{lccccc}
\hline$C=A \cap B$ & $A$ & $B$ & $A+B$ & Conclusion & Interpretation \\
\hline Lithology $\cap$ Road $=0.283$ & 0.154 & 0.135 & 0.289 & $C<A+B ; C>A, B$ & $\uparrow$ \\
Soil $\cap$ Road $=0.240$ & 0.120 & 0.135 & 0.255 & $C<A+B ; C>A, B$ & $\uparrow$ \\
Road $\cap$ Vegetable $=0.229$ & 0.135 & 0.088 & 0.223 & $C>A+B$ & $\uparrow \uparrow$ \\
Lithology $\cap$ Soil =0.210 & 0.154 & 0.120 & 0.274 & $C<A+B ; C>A, B$ & $\uparrow$ \\
Lithology $\cap$ Population $=0.210$ & 0.154 & 0.105 & 0.259 & $C<A+B ; C>A, B$ & $\uparrow$ \\
Lithology $\cap$ Settlement $=0.208$ & 0.154 & 0.073 & 0.227 & $C<A+B ; C>A, B$ & $\uparrow$ \\
Elevation $\cap$ Population $=0.208$ & 0.062 & 0.105 & 0.167 & $C>A+B$ & $\uparrow \uparrow$ \\
Lithology $\cap$ Elevation $=0.205$ & 0.154 & 0.062 & 0.216 & $C<A+B ; C>A, B$ & $\uparrow$ \\
Soil $\cap$ Vegetable $=0.205$ & 0.120 & 0.088 & 0.225 & $C<A+B ; C>A, B$ & $\uparrow$ \\
Road $\cap$ Population $=0.206$ & 0.135 & 0.105 & 0.240 & $C<A+B ; C>A, B$ & $\uparrow$ \\
\hline
\end{tabular}

“个” denotes $A$ and $B$ enhance each other; " $\uparrow$ " denotes a nonlinear enhancement of $A$ and $B$.

Table 6. Difference of mean deterioration KRD index between soil types.

\begin{tabular}{|c|c|c|c|c|c|c|c|c|}
\hline & $\begin{array}{c}\text { Calcareous } \\
\text { soil }\end{array}$ & Rendzina & $\begin{array}{l}\text { Purplish } \\
\text { soil }\end{array}$ & $\begin{array}{l}\text { Acidic } \\
\text { lithosol }\end{array}$ & $\begin{array}{c}\text { Skeletal } \\
\text { soil }\end{array}$ & $\begin{array}{l}\text { Paddy } \\
\text { soil }\end{array}$ & $\begin{array}{c}\text { Yellow } \\
\text { soil }\end{array}$ & $\begin{array}{l}\text { Terra } \\
\text { rossa }\end{array}$ \\
\hline \multicolumn{9}{|l|}{ Calcareous soil } \\
\hline Rendzina & $\mathrm{Y}$ & & & & & & & \\
\hline Purplish soil & $\mathrm{Y}$ & $\mathrm{Y}$ & & & & & & \\
\hline Acidic lithosol & $\mathrm{Y}$ & $\mathrm{Y}$ & $\mathrm{N}$ & & & & & \\
\hline Skeletal soil & $\mathrm{Y}$ & $\mathrm{Y}$ & $\mathrm{Y}$ & $\mathrm{Y}$ & & & & \\
\hline Paddy soil & $\mathrm{Y}$ & $\mathrm{Y}$ & $\mathrm{Y}$ & $\mathrm{Y}$ & $\mathrm{Y}$ & & & \\
\hline Yellow soil & $\mathrm{Y}$ & $\mathrm{Y}$ & $\mathrm{Y}$ & $\mathrm{Y}$ & $\mathrm{Y}$ & $\mathrm{Y}$ & & \\
\hline Terra rossa & $\mathrm{Y}$ & $\mathrm{Y}$ & $\mathrm{Y}$ & $\mathrm{Y}$ & $\mathrm{Y}$ & $\mathrm{Y}$ & $\mathrm{N}$ & \\
\hline
\end{tabular}

respectively) are still detected as the major driving factors of KRD deterioration in Changshun. The GDP and population density have low impacts on the spatial distribution of KRD deterioration. It seems that natural factors (with ranks of first, second, fifth, sixth, and seventh) have a relatively greater impact on KRD deterioration than anthropogenic factors (ranked third, fourth, eighth, and ninth).

\subsubsection{Variations of KRD deterioration at different driving factor levels}

The PD of soil, which has the highest ranking, shows that it is the most important of the driving factors influencing KRD deterioration. Table 6 shows significant differences in the mean deterioration KRD index between the eight soil types. The order of mean values of deterioration index for the eight soil types is as follows: acidic lithosol $(-57.0) \approx$ purplish soil $(-53.1)>$ terra rossa $(-36.6) \approx$ yellow soil $(-35.3)>$ paddy soil $(-33.3)>$ calcareous soil $(-28.7)>$ skeletal soil $(-23.8)>$ rendzina $(-15)$ (where “ $\approx$ " denotes that there is no significant difference between deterioration index of the two variables). This confirms that soil types are associated with their different hydrological proper ties and susceptibil- ity to erosion, which influence KRD transformation. Results suggest that the acidic lithosol and purplish soil significantly worsened KRD deterioration.

The influence of roads was again identified as being the most important of the anthropogenic factors influencing KRD deterioration. The order of mean values of the deterioration index for six road influence levels is as follows: level $2(-39.8)>$ level $6(-36.3)>$ level $1(-34.1)>$ level 4 $(-32.3)>$ level $5(-28.6)>$ level $3(-27.0)$. This shows that road influence is not monotonously correlated to the KRD deterioration intensities. Areas of levels 1 and 2, which are the nearest to roads, have severe KRD deterioration intensities. This indicates that human activities would enhance KRD deterioration. With distances to roads increasing, the areal deterioration intensity of levels 3, 4 and 5 decreases. However, the areal deterioration intensity at level 6 actually increases. An area with few human activities easily deteriorates, which may be attributed to other driving factors.

\subsubsection{Interactions of driving factors influencing KRD deterioration}

The top 10 PDs corresponding to interactive impacts on KRD deterioration are listed in Table 7; they range from 0.284 to 
Table 7. Interaction between pairs of driving factors influencing the KRD deterioration.

\begin{tabular}{lccccc}
\hline$C=A \cap B$ & $A$ & $B$ & $A+B$ & Conclusion & Interpretation \\
\hline Soil $\cap$ Lithology $=0.477$ & 0.194 & 0.151 & 0.345 & $C>A+B$ & $\uparrow \uparrow$ \\
Soil $\cap$ Settlement $=0.423$ & 0.194 & 0.14 & 0.334 & $C>A+B$ & $\uparrow \uparrow$ \\
Soil $\cap$ Road $=0.384$ & 0.194 & 0.143 & 0.337 & $C>A+B$ & $\uparrow \uparrow$ \\
Soil $\cap$ Elevation $=0.338$ & 0.194 & 0.138 & 0.332 & $C>A+B$ & $\uparrow \uparrow$ \\
Soil $\cap$ Vegetable $=0.336$ & 0.194 & 0.099 & 0.293 & $C>A+B$ & $\uparrow \uparrow$ \\
Road $\cap$ Settlement $=0.331$ & 0.143 & 0.14 & 0.283 & $C>A+B$ & $\uparrow \uparrow$ \\
Lithology $\cap$ Road =0.311 & 0.151 & 0.143 & 0.294 & $C>A+B$ & $\uparrow \uparrow$ \\
Settlement $\cap$ Elevation $=0.308$ & 0.14 & 0.138 & 0.278 & $C>A+B$ & $\uparrow \uparrow$ \\
Road $\cap$ Elevation $=0.288$ & 0.143 & 0.138 & 0.281 & $C>A+B$ & $\uparrow \uparrow$ \\
Lithology $\cap$ Elevation $=0.284$ & 0.151 & 0.138 & 0.289 & $C<A+B ; C>A, B$ & $\uparrow$ \\
\hline
\end{tabular}

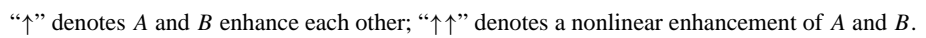

0.477. Except for the interactive impact of the lithology associated with elevation, the other interactive impacts of driving factors on KRD deterioration show non-linear enhancement (Table 7). For example, the PD of the soil associated with the lithology is 0.477 , far greater than their linear summation result (i.e., 0.345). Furthermore, the deterioration index of homogenous limestone/dolomite is minimized (i.e., -35.3) along with the deterioration of the acidic lithosol (i.e., -57.0 ). If the area corresponds to a region of homogenous limestone and acidic lithosol, then the interactive impact of high-permeability carbonate rocks, in terms of lithology and a low soil formation rate in soil types, would exacerbate the KRD deterioration.

\section{Discussion}

Using GIS techniques and the geographical detector model, our novel investigation has explored the available information at a much finer scale than previous studies (Huang and Cai, 2007; Jiang et al., 2009, 2014; Li et al., 2009a, b; Liu et al., 2008a; Xiong et al., 2009; Yan and Cai, 2013; Yang et al., 2013). Such studies omitted information about the spatial consistency of KRD evolution with respect to driving factors and also lacked consideration of interactions between various factors. We calculated the PDs of driving factors, which relate to the spatial consistency of driving factors compared with the E-KRD index. The order of the PDs is indicative of their relative importance to the evolution of KRD. Interactive PDs also enable the investigation of the combined impact of driving factors.

The influences of lithology, soil and roads are identified as the leading factors for KRD transformation in Changshun. Lithological types associated with the permeability of carbonate rocks and soil types associated with soil erosion and formation rates constitute the basic geographical environment that significantly affects the evolution of KRD (Jiang et al., 2014; Peng et al., 2013). Such influences suggest that in Changshun the design of restoration projects should consider the impacts of lithology and soil, and even more so their interactions. Our results confirm the important impact that human factors have on KRD. In particular, roads, as a proxy for human activity, have a significant influence as noted by their association with KRD improvement or deterioration. However, KRD deterioration in areas with a low road influence may be attributed to other driving factors. On one hand, better access to roads does enhance successful implementation of KRD restoration projects (Deng et al., 2011; Xu et al., 2013; Yang et al., 2011). On the other hand, however, when roads penetrate into (or are improved in) areas, they may intensify efforts to exploit resources (Deng et al., 2011) and therefore cause KRD deterioration (Mick, 2010; Yang et al., 2013). Road construction can create numerous roadcuts and embankments that can contribute to increased sediment production and further cause land degradation (Lee et al., 2013). Serious soil erosion can occur on bare road embankments (especially those under construction) (Cerdà, 2007) and on unpaved roads (Cao et al., 2013b). Restoration efforts should be directed to guarantee key ecological processes and support soil formation for roadcuts and embankments (Jimenez et al., 2013; Lee et al., 2013). New strategies should emphasize the need to decrease the impact of road construction on plant and animal habitats and the benefits of using local species for the re-vegetation of land surrounding the roads (Cheng et al., 2013).

Comparing the relative importance of natural and anthropogenic impact factors reveals some interesting findings. Previous studies have considered anthropogenic activities as being more significant in KRD than natural factors (Lan and Xiong, 2001; Li et al., 2009a; Yan and Cai, 2013; Yang et al., 2011). However, other studies have claimed that natural factors were the major factor in KRD evolution (Gu et al., 2011; Hu et al., 2004; Shan, 2006). In our case study, there is no significant difference observed between the impacts of natural and anthropogenic factors influencing the KRD improvement based on the order of their PDs (Table 8). Even natural factors have a higher impact on KRD deterioration. In fact it could 
Table 8. PDs of driving data and their order for both the KRD improvement and deterioration.

\begin{tabular}{lcccc}
\hline & \multicolumn{2}{c}{ KRD improvement } & \multicolumn{2}{c}{ KRD deterioration } \\
& PD & Order & PD & Order \\
\hline Soil type & 0.120 & 3 & 0.194 & 1 \\
Lithology & 0.154 & 1 & 0.151 & 2 \\
Vegetation type & 0.088 & 5 & 0.099 & 6 \\
Elevation & 0.062 & 7 & 0.138 & 5 \\
Slope & 0.029 & 8 & 0.056 & 7 \\
Road influence & 0.135 & 2 & 0.143 & 3 \\
Settlement influence & 0.073 & 6 & 0.140 & 4 \\
GDP density & 0.013 & 9 & 0.022 & 8 \\
Population & 0.105 & 4 & 0.014 & 9 \\
\hline
\end{tabular}

be argued that natural factors have a greater impact on KRD deterioration. The scaling effect would lead to different results for KRD driving factors in different spatial and temporal scales (Yang et al., 2009), and may cautiously support the importance of anthropogenic driving factors in KRD. However, there is little unassailable evidence to support whether or not natural or anthropogenic factors have the greater impact. In the field survey, a significant contrast in adjacent regions with carbonate rock and non-carbonate rocks (granite) can be found, but under nearly the same conditions of anthropogenic factors. There is an obvious KRD in areas with the carbonate rock outcropping. In contrast, areas with noncarbonate rocks are covered with lush vegetation. Furthermore, a historical study showed that the spatial distribution of suspect KRD in Guizhou in 1940 was similar to the situation in 2005 (Han et al., 2011). Within this stage of rapid population growth, the spatial distribution stays relatively stable. Above evidence indicated that we cannot overemphasize the impact of anthropogenic factors. Karst is an integrated and unique geo-ecosystem, and its evolution is caused by natural and human factors (Febles-González et al., 2012). KRD occurs under a specific karst background, and anthropogenic factors can aggravate or reverse the KRD in a relatively short period, but may not be the essential driving factor (Zhang and Zhou, 2001). Without the specific environments, informal human activities cannot cause KRD (Shan, 2006).

Although slope angles have a significant influence on KRD (Huang and Cai, 2007; Jiang et al., 2009), this impact factor was ranked quite low for both KRD improvement and deterioration (the PDs rank eighth and seventh respectively) in our study. Soil on steepened slopes is often susceptible to erosion, which is one of the reasons leading to KRD (Ying et al., 2014). Therefore, areas of KRD are mainly located in sloping regions in Changshun. Also, the mean KRD improvement index with small slope angles is greater than that with large slope angles. However, local areas with steepened slopes can also be highly vegetated in our field survey, which may be attributed to the fact that high slope angles limit the range of possible human activities and thereby act to reduce anthropogenic disturbances (Xu et al., 2013). Areas of low- slope angles (especially $<5^{\circ}$ ) may be suitable for agricultural use which then can lead to KRD (Huang and Cai, 2007). Therefore, slope angle might not play a significant role in soil erosion of karst regions (Peng et al., 2013). The complex effects of slope cause an inconsistent spatial distribution of the KRD transformation versus slope angles.

Until now, few studies have investigated the interactive effects of driving factors on KRD transformations. Our results show that those interactive factors lead to enhanced impacts. Major interactive results of the KRD improvement conclude that "PD $(A \cap B)<\mathrm{PD}(A)+\mathrm{PD}(B)$ " (Table 5), whereas the major results of the KRD deterioration conclude that "PD $(A \cap B)>\mathrm{PD}(\mathrm{A})+\mathrm{PD}(B)$ " (Table 7). The enhanced effect on KRD deterioration is more significant than for KRD improvement. Compared with the impact of a single factor, the nonlinearly enhanced effects of interactive factors on KRD transformations should be explored in the further studies to effectively characterize KRD evolution. For example, the interactive impact of lithology associated with soil on KRD deterioration and road influence associated with vegetation on KRD improvement should be considered in the design of restoration projects.

There are some uncertainties in our study. Human activities, such as farming on hilly lands, overgrazing, and felling of forest and shrubs for cooking (Jiang et al., 2014; Li et al., 2009b; Liu et al., 2008a; Wu et al., 2011; Yan and Cai, 2013), are rather difficult to measure in a straightforward manner and would not be depicted at a detailed spatial distribution. Instead, they were represented by proxies of distances to roads and settlements in our study, which have proven to be acceptable in recent studies. Moreover, summing PDs of driving data ( 0.779 for improvement and 0.957 for KRD deterioration), it indicates that our selected driving data have a relatively larger impact on KRD deterioration than improvement. Indeed, we could not include all the driving data in the study. For example, meteorological factors are relatively similar at the county scale and were neglected in this study. However, the impact of frequent droughts and extreme floods induced by global climate change on the KRD transformation should be investigated further (Gu et al., 2011; Huang et al., 
2009; Jiang et al., 2014). It would be interesting to explore the significance of meteorological factors and their interactions with other factors in a macro scale study.

\section{Conclusions}

A comprehensive exploration of driving factors influencing KRD evolution can provide the information needed to effectively combat further deterioration in a fragile karst environment. We use GIS techniques to quantify the information on human activity farming (hilly lands, overgrazing, felling and restoration projects) by proxies of distances to roads and settlements for it is difficult to measure it directly. We used a geographical detector model to incorporate fine-scale spatial information in our investigation of the relationships between driving factors and KRD transformation in Changshun. The orders of PDs for natural and anthropogenic factors show the variable impact that they have on the spatial distribution of KRD improvement or deterioration. Lithology, soil and road influence are identified as the major driving factors associated with KRD evolution. Our results imply that there is no significant difference between natural and anthropogenic factors when it comes to influencing KRD improvement, but natural factors have a higher impact on KRD deterioration. The specifics of the karst background create a fragile and vulnerable environment that is susceptible to human activities, and then anthropogenic factors significantly influence KRD transformation further. There is little unassailable evidence to support whether or not the natural or anthropogenic factors have a greater impact. However, we did detect the impact of interactive factors and found that non-linearly enhanced impacts significantly aggravated the KRD. Our results have explored new information concerning the relevant influence on KRD evolution, which can help to effectively restore KRD. The limitations and improvements mentioned above should be explored in future studies.

Acknowledgements. This work was jointly supported by the National Basic Research Program of China (973 Program) (2015CB452702), the National Program of Monitoring Desertification sponsored by the Combating Desertification Management Center of the State Forestry Administration, and the Program of Regional Sustainable Land Use sponsored by the Ministry of Land and Resources. We are most grateful for the suggestion of the geographical detectors model by Xu Chengdong and Ren Zhoupeng.

Edited by: A. Cerdà

\section{References}

Bai, X. Y., Wang, S. J., and Xiong, K. N.: Assessing spatialtemporal evolution processes of karst rocky desertification land: indications for restoration strategies, Land Degrad. Dev., 24, 15 47-56, 2013.
Cao, F., Ge, Y., and Wang, J. F.: Optimal discretization for geographical detectors-based risk assessment, GISci. Remote Sens., 50, 78-92, 2013a.

Cao, L., Zhang, K., Dai, H., and Liang, Y.: Modeling interrill erosion on unpaved roads in the loess plateau of China, Land Degrad. Dev., doi:10.1002/ldr.2253, 2013b.

Cerdà, A.: Soil water erosion on road embankments in eastern Spain, Sci. Total Environ., 378, 151-155, 2007.

Cheng, B., Lv, Y., Zhan, Y., Su, D., and Cao, S.: Constructing China's roads as works of art: a case study of "esthetic greenway" construction in the Shennongjia region of China, Land Degrad. Dev.,doi:10.1002/ldr.2210, 2013.

Deng, X. Z., Huang, J. K., Huang, Q. Q., Rozelle, S., and Gibson, J.: Do roads lead to grassland degradation or restoration?, A case study in Inner Mongolia, China, Environ. Dev. Econ., 16, 751773, 2011.

Febles-González, J., Vega-Carreño, M., Tolón-Becerra, A., and Lastra-Bravo, X.: Assessment of soil erosion in karst regions of Havana, Cuba, Land Degrad. Dev., 23, 465-474, 2012.

Gu, X. P., Yu, F., Liu, Y. H., and Luo, Y. X.: Impact of precipitation factor on rocky desertification in karst areas, Bull. Soil Water Conservat., 31, 66-70, 2011 (in Chinese).

Han, Z. Q. and Yang, S. C. : A comparative study of the distribution of suspect karst rocky desertification recorded in the archives from the 1940s and that from today's survey, Journal of Chinese Historical Geography, 26, 32-40, 2011 (in Chinese).

Hu, B. Q., Liao, C. M., Yan, Z. Q., Jiang, S. F., Huang, Q. Y., and Li, S. M.: Diving mechanism diagnosis of karst rocky desertification in Duan Yao Autonomous County of Guangxi based on RS and GIS, J. Mt. Sci., 22, 583-590, 2004 (in Chinese).

Hu, Y., Wang, J. F., Li, X. H., Ren, D., and Zhu, J.: Geographical detector-based risk assessment of the under-five mortality in the 2008 Wenchuan Earthquake, China, PloS one, 6, e21427, doi:10.1371/journal.pone.0021427, 2011.

Huang, Q. H. and Cai, Y. L.: Spatial pattern of karst rock desertification in the middle of Guizhou Province, Southwestern China, Environ. Geol., 52, 1325-1330, 2007.

Huang, Y. Q., Zhao, P., Zhang, Z. F., Li, X. K., He, C. X., and Zhang, R. Q.: Transpiration of Cyclobalanopsis glauca (syn. Quercus glauca) stand measured by sap-flow method in a karst rocky terrain during dry season, Ecol. Res., 24, 791-801, 2009.

Jiang, Y., Li, L., Groves, C., Yuan, D., and Kambesis, P.: Relationships between rocky desertification and spatial pattern of land use in typical karst area, Southwest China, Environ. Earth Sci., 59, 881-890, 2009.

Jiang, Z. C., Lian, Y. Q., and Qin, X. Q.: Rocky desertification in Southwest China: impacts, causes, and restoration, Earth-Sci. Rev., 132, 1-12, 2014.

Jimenez, M., Ruiz-Capillas, P., Mola, I., Pérez-Corona, E., Casado, M., and Balaguer, L.: Soil development at the roadside: a case study of a novel ecosystem, Land Degrad. Dev., 24, 564-574, 2013.

Lan, A. J. and Xiong, K. N.: Analysis on driving factors of karst rock-desertification - with a special reference to guizhou province, Bull. Soil Water Conservat., 21, 19-23, 2001.

Lee, J. W., Park, C. M., and Rhee, H.: Revegetation of decomposed granite roadcuts in Korea: developing digger, evaluating cost effectiveness, and determining dimensions of drilling holes, reveg- 
etation species, and mulching treatment, Land Degrad. Dev., 24, 591-604, 2013.

Li, L. F., Wang, J. F., Cao, Z. D., and Zhong, E. S.: An informationfusion method to identify pattern of spatial heterogeneity for improving the accuracy of estimation, Stoch. Env. Res. Risk A., 22, 689-704, 2008.

Li, S., Wang, J. H., Wang, X. Z., Li, H. X., Chen, Z. J., and Liao, X. $X$. : A study of rocky desertification processes and their driving forces in northern part of Guangdong China, from 1974 to 2004 - a case study on four counties of Yingde, Yangshan, Ruyuan and Lianzhou, J. Nat. Resour.s, 24, 816-826, 2009 (in Chinese).

Li, X. W., Xie, Y. F., Wang, J. F., Christakos, G., Si, J. L., Zhao, H. N., Ding, Y. Q., and Li, J.: Influence of planting patterns on fluoroquinolone residues in the soil of an intensive vegetable cultivation area in northern China, Sci. Total Environ., 458, 63-69, 2013.

Li, Y. B., Shao, J. A., Yang, H., and Bai, X. X.: The relations between land use and karst rocky desertification in a typical karst area, China, Environ. Geol., 57, 621-627, 2009.

Liu, Y. S., Wang, J., and Deng, X.: Rocky land desertification and its driving forces in the karst areas of rural Guangxi, Southwest China, J. Mt. Sci., 5, 350-357, 2008a.

Liu, Y. S., Wang, J. Y., and Deng, X. Z.: Rocky land desertification and its driving forces in the karst areas of rural Guangxi, Southwest China, J. Mt. Sci., 5, 350-357, 2008b.

Mick, D.: Human interaction with Caribbean karst landscapes: past, present and future, Acta Carsologica, 39, 137-146, 2010.

Peng, J., Xu, Y. Q., Zhang, R., Xiong, K. N., and Lan, A. J.: Soil erosion monitoring and its implication in a limestone land suffering from rocky desertification in the Huajiang Canyon, Guizhou, Southwest China, Environ. Earth Sci., 69, 831-841, 2013.

Peng, T. and Wang, S. J.: Effects of land use, land cover and rainfall regimes on the surface runoff and soil loss on karst slopes in southwest China, Catena, 90, 53-62, 2012.

Qi, X. K., Wang, K. L., and Zhang, C. H.: Effectiveness of ecological restoration projects in a karst region of southwest China assessed using vegetation succession mapping, Ecol. Eng., 54, 245-253, 2013.

Shan, Y. T.: Karst rocky desertification and analysis on the geological fators in Southwest China, Carsologica Sinica, 25, 163-167, 2006 (in Chinese).

Sheng, M. Y., Liu, Y., and Xiong, K. N.: Response of soil physicalchemical properties to rocky desertiffication succession in South China Karst, Acta Ecologica Sinica, 33, 6303-6313, 2013 (in Chinese).

Shi, X. Z., Yu, D. S., Warner, E. D., Pan, X. Z., Petersen, G. W., Gong, Z. G., and Weindorf, D. C.: Soil Database of $1: 1000000$ Digital Soil Survey and Reference System of the Chinese Genetic Soil Classification System, Soil Surv. Horizons 45, 129136, 2004..

Simpson, R. D. and Christensen, N. L.: Ecosystem Function and Human Activities: Reconciling Economics and Ecology, Chapman \& Hall, 1997.

Solutions, I. V. I.: Atmospheric Correction Module: QUAC and FLAASH User's Guide, 2009.

Wang, J. F., Haining, R., and Cao, Z. D.: Sample surveying to estimate the mean of a heterogeneous surface: reducing the error variance through zoning, Int. J. Geogr. Inf. Sci., 24, 523-543, 2010a.
Wang, J. F., Li, X. H., Christakos, G., Liao, Y. L., Zhang, T., Gu, X., and Zheng, X. Y.: Geographical detectors-based health risk assessment and its application in the neural tube defects study of the Heshun Region, China, Int. J. Geogr. Inf. Sci., 24, 107-127, 2010b.

Wang, S. J., Li, R. L., Sun, C. X., Zhang, D. F., Li, F. Q., Zhou, D. Q., Xiong, K. N., and Zhou, Z. F.: How types of carbonate rock assemblages constrain the distribution of karst rocky desertified land in Guizhou Province, PR China: phenomena and mechanisms, Land Degrad. Dev., 15, 123-131, 2004a.

Wang, S. J., Liu, Q. M., and Zhang, D. F.: Karst rocky desertification in southwestern China: geomorphology, landuse, impact and rehabilitation, Land Degrad. Dev., 15, 115-121, 2004 b.

Wang, X. Y.: Comprehensive treatment of rocky desertification in karst mountain areas, Chin. J. Agr. Resour. Reg. Plann., 31, 7680, 2010 (in Chinese).

Wu, X. Q., Liu, H. M., Huang, X. L., and Zhou, T.: Human driving forces: analysis of rocky desertification in karst region in Guanling County, Guizhou Province, Chinese Geogr. Sci., 21, 600608, 2011.

Xiong, Y. J., Qiu, G. Y., Mo, D. K., Lin H, Sun H, Wang, Q. X., Zhao, S. H., and Yin, J.: Rocky desertification and its causes in karst areas: a case study in Yongshun County, Hunan Province, China, Environ. Geol., 57, 1481-1488, 2009.

Xu, E. Q., Zhang, H. Q., and Li, M. X.: Mining spatial information to investigate the evolution of karst rocky desertification and its human driving forces in Changshun, China, Sci. Total Environ., 458, 419-426, 2013.

Yan, X. and Cai, Y. L.: Multi-scale anthropogenic driving forces of karst rocky desertification in southwest China, Land Degrad. Dev., doi:10.1002/ldr.2209, 2013.

Yang, Q. Q., Wang, K. L., and Yue, Y. M.: Spatial distribution of rocky desertification and its difference between scales in northwest Guangxi, Ac. Ecol. Sinica, 29, 3629-3640, 2009 (in Chinese).

Yang, Q. Q., Wang, K. L., Zhang, C. H., Yue, Y. M., and Tian, R. C.: Spatio-temporal evolution of rocky desertification and its driving forces in karst areas of Northwestern Guangxi, China, Environ. Earth Sci., 64, 383-393, 2011.

Yang, Q. Y., Jiang, Z. C., Ma, Z. L., Luo, W. Q., Xie, Y. Q., and Cao, J. H.: Relationship between karst rocky desertification and its distance to roadways in a typical karst area of Southwest China, Environ. Earth Sci., 70, 295-302, 2013.

Ying, B., Xiao, S. Z., Xiong, K. N., Cheng, Q. W., and Luo, J. S.: Comparative studies of the distribution characteristics of rocky desertification and land use/land cover classes in typical areas of Guizhou province, China, Environ. Earth Sci., 71, 631-645, 2014.

Zeng, F. P., Peng, W. X., Song, T. Q., Wang, K. L., Wu, H. Y., Song, X. J., and Zeng, Z. X.: Changes in vegetation after 22 years' natural restoration in the Karst disturbed area in northwestern Guangxi, China, Ac. Ecol. Sinica, 27, 5110-5119, 2007.

Zhang, D. F. and Zhou, D. Q.: Intrinsic driving mechanism of land rocky desertification in karst regions of Guizhou Province, Bull. Soil Water Conservat., 21, 1-5, 2001 (in Chinese).

Zhou, M. W., Wang, S. J., and Li, Y. B.: Spatial factor analysis of karst rocky desertification landscape patterns in Wangjiazhai catchment, Guizhou, Geogr. Res., 26, 897-905, 2007. 